\title{
Transcranial magnetic stimulation in clinical practice
}

\author{
Sheila Hardy, Lorraine Bastick, Alex O’Neill-Kerr, Priyadharshini Sabesan, \\ Sudheer Lankappa \& Lena Palaniyappan
}

\begin{abstract}
SUMMARY
Up to $40 \%$ of people with depression do not recover following standard treatments such as medication and psychotherapy. Transcranial magnetic stimulation (TMS) is a treatment used worldwide for adult patients with severe clinical depression when antidepressants have repeatedly failed to control their symptoms. This article explains the use of TMS in clinical practice.
\end{abstract}

\section{LEARNING OBJECTIVES}

- Understand how TMS works in the treatment of depression

- Appreciate which patients will benefit from TMS and when it is contraindicated

- Be aware of the current clinical guidance for the use of TMS as a treatment for depression

\section{DECLARATION OF INTEREST}

L.P. received travel support to speak at a meeting organised by Magstim (makers of a TMS device) at Oxford in 2014.

The American Psychiatric Association (APA; 2013) describes major depressive disorder as a medical illness that affects how a person feels, thinks and behaves, causing persistent feelings of sadness and loss of interest in previously enjoyed activities. It can lead to a variety of emotional and physical problems and usually requires long-term treatment. However, between 20 and $40 \%$ of people with depression do not recover following standard treatments such as medication and psychotherapy (Bauer 2002).

Transcranial magnetic stimulation (TMS), a form of neuromodulation, is a non-invasive, nonconvulsive technique used to stimulate neural tissue. The procedure involves the placement of an electromagnetic coil to deliver a short, powerful magnetic field pulse (1.5-2.0 T) through the scalp and induce electric current in the brain. TMS is a relatively new treatment for psychiatric disorders, but it has been well established in neuroscience research experiments and in clinical applications for neurological conditions. Additionally, it has been used as an investigative tool in neuronal diseases (Groppa 2012). Box 1 summarises the applications of TMS.

\section{Treatment}

Barker and his colleagues in Sheffield first demonstrated that TMS could be applied to stimulate the cerebral cortex (Barker 1985). The stimulation can be delivered either as a single pulse or as repetitive pulses (repetitive TMS or rTMS). The clinical use of TMS involves repetitive pulses applied in trains of stimulation over a target area of the cortical region of the brain. The response to the treatment is influenced by various factors, including the frequency, intensity and duration of the stimulus, and its ability to excite or inhibit particular neuronal functions (Hoogendam 2010). The strength of the magnetic field used for clinical applications is similar to that of a standard magnetic resonance imaging (MRI) scanner (Rossi 2009).

\section{Putative mechanism of action}

As is the case for many other antidepressant treatments that are available in clinical practice, the exact mechanism by which rTMS produces relief from depression is still unknown. At a physiological level, the effects of rTMS are frequently reported to be similar to long-term potentiation (LTP) or long-term depression (LTD)

BOX 1 What is TMS and when is it used?

- Transcranial magnetic stimulation (TMS) is the application of magnetic pulses over the scalp to produce electrical activity in specific regions of the brain

- In repetitive transcranial magnetic stimulation (rTMS) treatment, the electromagnetic coil is switched on and off repeatedly to produce stimulating pulses

- The main indication for TMS treatment is unipolar treatment-resistant depression without psychotic symptoms, especially in patients who do not want to consider electroconvulsive therapy (ECT)

- Treatment of depression using TMS usually involves daily sessions 4 or 5 days a week for $4-5$ weeks

\section{ARTICLE}

Sheila Hardy is an Education Fellow at UCLPartners, an Honorary Senior Lecturer at UCL and a Visiting Fellow at the University of Northampton. Lorraine Bastick is at the Northamptonshire Healthcare NHS Foundation Trust. Alex O'Neill-Kerr is the Medical Director for the Northamptonshire Healthcare NHS Foundation Trust. Priyadharshini Sabesan is at the Nottinghamshire Healthcare Foundation Trust. Sudheer Lankappa is the Clinical Lead for the Nottingham Neuromodulation Unit, Queen's Medical Centre, Nottingham. Lena Palaniyappan is an Associate Professor in the Departments of Psychiatry and Medical Biophysics at the University of Western Ontario.

Correspondence Dr Sheila Hardy, Northamptonshire Healthcare NHS Foundation Trust, Berrywood Hospital, Berrywood Drive, Northampton NN5 6UD, UK. Email: sheila.hardy@nhft.nhs.uk 
of stimulated neurons. This, in turn, implicates changes in synaptic plasticity (that is, the ability of synapses to strengthen or weaken over time in response to increases or decreases in their activity) (Fitzgerald 2006). LTP and LTD are two forms of long-term synaptic plasticity that result in a longlasting experience-dependent change in the efficacy of synaptic transmission (that is, the amplitude of synaptic potentials). LTP and LTD have been widely studied as they represent cellular correlates of learning and memory (Luscher 2012). However, invoking this mechanism to explain the effects of rTMS is oversimplistic; further exploration is therefore required (Hoogendam 2010). A number of studies indicate that large-scale brain networks are altered in patients with depression, and that the degree of the change in the connectivity of these networks predicts the severity of the depression (Salomons 2014). Repetitive stimulation of the focal nodes of these networks can result in reorganisation of connectivity patterns in the brain, owing to the brain's inherent plasticity, and use-dependent strengthening of existing pathways (Box 2). Research suggests that rTMS does indeed result in changes in regional brain activity and metabolism, and that applying rTMS at the left dorsolateral prefrontal cortex (DLPFC) can enhance this region's connectivity with other regions that are crucial for regulating emotional processing (Kito 2008).

\section{Clinical guidance and approvals}

Worldwide, rTMS is being used as a treatment option for depressive disorders. In 2008, the US Food and Drug Administration (FDA) approved a TMS device for treatment of patients with depression (Horvath 2010). This approval was based on results from one study of patients who had failed to respond to one previous antidepressant. The APA, the Royal Australian and New Zealand College of Psychiatrists (RANZCP), the British Association of Psychopharmacology (BAP) and organisations in various European countries have discussed the state of the evidence for the efficacy of rTMS in their depression management guidelines and when rTMS might be considered.

\section{BOX 2 How rTMS works}

- Stimulates specific regions of the cerebral cortex

- Possibly improves the efficacy of synaptic transmission

- Repetitive stimulation of focal nodes of brain networks may result in reorganisation of their connectivity patterns that regulate emotional processing
In England, the National Institute for Health and Care Excellence (NICE) issued guidance for the use of rTMS, recommending that its use as a treatment for depression should be restricted to research settings until optimal dosing and application parameters are established (NICE 2007). This guidance was reviewed and updated in 2011; however, the recommendation regarding TMS was not changed, owing to lack of evidence. However, in 2012, NHS Evidence reviewed newer research, including a comprehensive systematic review (Allan 2011). They concluded that rTMS may be a therapeutic option for patients with treatmentresistant depression as part of specialist team management. They also highlighted that further research is needed to define optimal treatment protocols. NICE completed a consultation in July 2015 (NICE 2015a). Updated guidance in December 2015 stated that rTMS for depression may be used with normal arrangements for clinical governance and audit (NICE 2015b).

\section{Indications}

At present, rTMS is indicated for depression when the patient is responding inadequately to conventional treatments (antidepressant medication, psychological therapy or a combination of these) and when electroconvulsive therapy (ECT) would be the next option.

Real-world data from 42 TMS clinics in North America indicate that the average number of suitable antidepressant treatment trials for a defined episode of depression undergone by patients who receive TMS is 2.6 (s.d. = 2.4) (Dunner 2014). A meta-analysis found ECT to be better than rTMS in terms of remission rates (52\% v. 34\%) and improvement in depressive symptoms, but similar in relation to drop-out rates (Berlim 2013a). The authors suggest that future comparative trials with larger sample sizes, better matching at baseline, longer follow-ups and more intense stimulation protocols are warranted. More recently, another systematic review (Ren 2014) comparing TMS and ECT concluded that ECT was superior to high-frequency rTMS in terms of response $(64.4 \%$ v. $48.7 \%$; risk ratio $(\mathrm{RR})=1.41$, $P=0.03)$ and remission $(52.9 \%$ v. 33.6\%; $\mathrm{RR}=1.38, P=0.006)$, whereas discontinuation was not significantly different between the two treatments $(8.3 \%$ v. $9.4 \%$; $\mathrm{RR}=1.11, P=0.80)$. This is essentially the same as the findings of Berlim et al, 2013a,b,c. There are insufficient data to draw any conclusions regarding the long-term efficacy of rTMS. One study has shown that patients with bipolar depression respond less well than those with unipolar depression (Connolly 2012), but 
there are no studies that specifically focus on the management of bipolar disorder with TMS. In summary, the main indication for TMS treatment is unipolar treatment-resistant depression without psychotic symptoms, especially in patients who do not want to consider ECT (Box 3).

At present, there is almost no evidence for the efficacy of rTMS in relapse prevention in depressive disorder. When using rTMS following a short-term response or remission from depression, it is important to continue optimal evidencebased pharmacotherapy and/or psychotherapeutic approaches aimed at preventing further relapses. rTMS, as indicated in Fig. 1, has no role in maintenance treatment for depression.

\section{Contraindications}

There are a number of contraindications for rTMS as a treatment for depression. These include:

- a history of epilepsy or organic brain pathology

- sleep deprivation

- acute alcohol-dependence syndrome

- use of drugs that cause a significant reduction in seizure threshold

- severe or recent heart disease

- presence of surgically placed ferromagnetic material such as a cochlear implant or cardiac pacemaker.

However, recent evidence suggests that sleep deprivation need not be considered to be a contraindication (Krstic 2014; Tang 2015). All patients should be screened using a standard questionnaire before being accepted for rTMS treatment.

\section{Patient selection}

As part of the process of selecting patients for rTMS, it is important to gain information on the clinical features of past treatment failures (Fitzgerald 2013). The latest interventional procedure guidance for rTMS in depression (NICE 2015) states that: 'The Committee was advised that the procedure may not be appropriate for treating some kinds of depression and that patient selection is therefore most important'. At present, most evidence supporting the use of rTMS has been gathered from randomised controlled trials (RCTs) involving out-patients. These patients had depression of sufficient severity to interfere with daily functioning, but their symptoms were not so severe as to prevent daily attendance on an out-patient basis to receive 2-3 weeks of rTMS treatment. Additionally, although many trials included patients who had failed to respond to at least two antidepressant treatments, the relationship between the degree of treatment
BOX 3 Clinical guidance and approval for rTMS

- Most guidelines acknowledge a role for rTMS in treating depression, but highlight the lack of robust evidence from large treatment-resistant samples

- New guidance from NICE in December 2015 states that rTMS for depression may be used with normal arrangements for clinical governance and audit

resistance and the effectiveness of rTMS continues to be unclear - especially as there is no unanimous, categorical definition of treatment-resistant depression (Mitchell 2006; Schutter 2009). rTMS is less effective than ECT in the presence of psychotic depression, although this observation is based on post hoc analyses from only a small number of head-to-head comparisons between the two techniques (Ren 2014).

\section{Overview of TMS administration}

Administration of rTMS is an out-patient procedure and does not require sedation or general anaesthesia. Once the patient is comfortably seated in a chair, a small insulated electromagnetic coil is placed on their scalp. In people with depression, placement of the coil is on the left DLPFC. The patient will have earplugs to mask the noise from the discharging coil. The intensity of rTMS is usually set as a percentage of the patient's motor threshold, which is defined as the minimum stimulus strength required to induce minimal

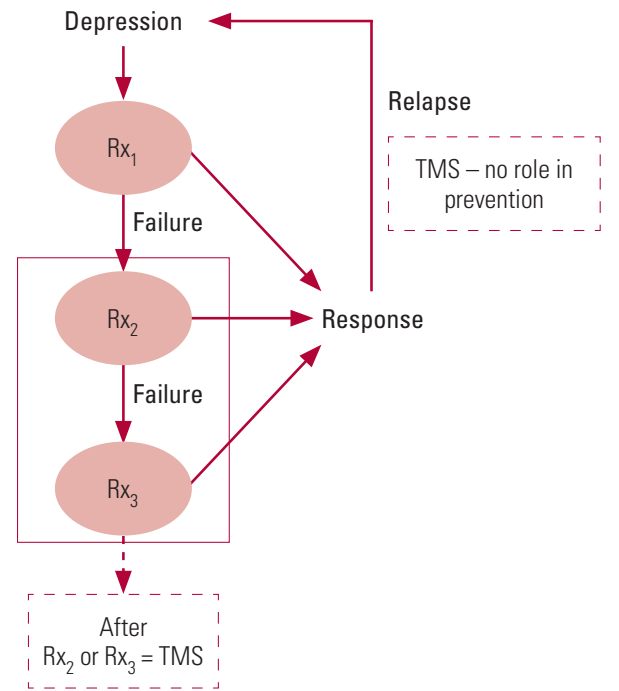

The place for transcranial magnetic stimulation (TMS) in the current depression treatment pathway. TMS may be used if the patient has not responded to two or more trials of antidepressant medication ( $\mathrm{Rx}$ ), but it is not appropriate for maintenance treatment or to prevent relapses. 
involuntary muscle movements (usually in the hand). Although there are various treatment protocols, the FDA-based standard parameters are the most widely used. These include an intensity of $120 \%$ of the patient's motor threshold, with a total of 3000 pulses per session, lasting $37.5 \mathrm{~min}(4 \mathrm{~s}$ of treatment once in every $26 \mathrm{~s}$ ). Treatment with rTMS usually involves daily sessions 5 days a week for 4-5 weeks and possibly longer (Horvath 2010).

\section{Efficacy}

The efficacy of rTMS treatment for major depressive disorder has been well established over recent years, using various different clinical populations as well as differing forms of TMS (high frequency, low frequency and bilateral) (Table 1). Many systematic reviews and meta-analyses of randomised, double-blind and sham-controlled trials of rTMS for treating major depressive disorder

TABLE 1 Meta-analytic studies of the antidepressant efficacy of repetitive transcranial magnetic stimulation (rTMS) published in or after $2003^{\text {a }}$

\begin{tabular}{|c|c|c|c|c|}
\hline Reference & Trials, $n$ & $\begin{array}{l}\text { rTMS sessions: } \\
\text { mean (s.d.) }\end{array}$ & $\begin{array}{l}\text { Participant age, } \\
\text { years: mean (s.d.) }\end{array}$ & Effect size \\
\hline Allan et al(2011) & 25 & Not presented & Not presented & $\begin{array}{l}\mathrm{NNT}=5 \mathrm{v} . \text { sham for EOT response, irrespective of site, overall dose, } \\
\text { treatment duration and antidepressant use }\end{array}$ \\
\hline Berlim et al (2013a) & 7 & $12.9(2.7)$ & $49.3(5.7)$ & $\begin{array}{l}\text { NNT }=6 \text { (EOT response from } 7 \text { RCTs using bilateral rTMS); NNT =7 (EOT } \\
\text { remission from } 4 \text { RCTs) } v \text {. sham }\end{array}$ \\
\hline Berlim et al (2013b) & 29 & $13.4(5.7)$ & $47.6(7.1)$ & $\begin{array}{l}\text { NNT }=6 \text { (EOT response from } 29 \text { RCTs using HF rTMS); } 8 \text { (EOT remission } \\
\text { from } 15 \text { RCTs); no difference in efficacy between studies with patients } \\
\text { having }<2 \mathrm{v} .>2 \text { antidepressant failures }\end{array}$ \\
\hline Berlim et al(2013c) & 8 & $12.6(3.9)$ & $49.39(7.0)$ & $\begin{array}{l}\mathrm{NNT}=5 \text { (EOT response from } 8 \text { RCTs using LF rTMS); NNT =5 (EOT } \\
\text { remission from } 6 \text { RCTs) }\end{array}$ \\
\hline Couturier (2005) & 6 & Not presented & Not presented & WMD = 1.1 (HF $\geq 10 \mathrm{~Hz}$ only included) \\
\hline Gaynes et al(2014) & 18 & Not presented & Not presented & $\begin{array}{l}\text { NNT = } 9 \text { (EOT response for TRD with } 2 \text { or more antidepressant failures } \\
\text { from } 5 \text { largest RCTs of } 14 \text { eligible); } 5 \text { (remission for TRD with } 2 \text { or more } \\
\text { antidepressant failures from } 7 \text { RCTs) irrespective of site, overall dose, } \\
\text { duration and severity }\end{array}$ \\
\hline Gross et al (2007) & 5 & $12.2(3.03)$ & $44.7(4.2)$ & $d=0.76$ for 2-week (EOT) change in severity (5 RCTs published in 2006) \\
\hline Herrmann \& Ebmeier (2006) & 33 & Not presented & $\begin{array}{l}49.14^{\text {b }} \text { (subgroups } \\
\text { split at age } 50)\end{array}$ & $\begin{array}{l}d=0.65 \text { for EOT mean change in severity, irrespective of age, TRD } \\
\text { status, dose and antidepressant use, based on } 33 \text { RCTs }\end{array}$ \\
\hline Kedzior et al (2014) & 14 & Not presented & $27.0-53.0^{c}$ & $\begin{array}{l}d=0.42 \text { based on } 14 \text { RCTs published between } 2010 \text { and 2013; } 8 \\
\text { included patients with } 2 \text { or more antidepressant failures; effect } \\
\text { irrespective of medication status and frequency of rTMS }\end{array}$ \\
\hline Lam et al (2008) & 24 & Not presented & Not presented & $\begin{array}{l}\text { NNT }=6 \text { for EOT response ( } 22 \text { RCTs); NNT }=7 \text { for EOT remission (16 } \\
\text { RCTs); NNT =6 for both EOT response ( } 14 \text { RCTs) and remission ( } 10 \text { RCTs) } \\
\text { with } 2 \text { or more antidepressant failures }\end{array}$ \\
\hline Lepping et al(2014) & 22 & Not presented & Not presented & $\begin{array}{l}d=0.63 \text { (EOT mean change for } 22 \text { RCTs for non-refractory depression; } \\
\text { equates to NNT = } 4 \text { according to Furukawa, 2014) }\end{array}$ \\
\hline Lepping et al (2014) & 10 & Not presented & Not presented & $\begin{array}{l}d=0.74 \text { (EOT mean change for } 10 \text { RCTs for inconsistently defined } \\
\text { 'refractory depression') }\end{array}$ \\
\hline Martin et al (2003) & 14 & Not presented & $41.8-60.9^{c}$ & $\begin{array}{l}d=0.35 \text { for } 2 \text {-week mean change in severity; } d=0.18 \text { (not significant) for } \\
1 \text {-week change (13 RCTs using HF rTMS) }\end{array}$ \\
\hline Schutter (2009) & 30 & $10.67(3.9)$ & $49.5(7.8)$ & $\begin{array}{l}d=0.39 \text { for EOT mean change in severity, irrespective of treatment } \\
\text { resistance and rTMS intensity (30 RCTs using HF rTMS) }\end{array}$ \\
\hline Berlim (2013d) & 6 & $13.3(4.08)$ & $44.47(7.55)$ & $\begin{array}{l}\text { NNT }=7 \mathrm{v} \text {. sham for response to HF rTMS at the end of use as add-on to } \\
\text { medications (on average } 3 \mathrm{rd} \text { week); NNT =5 for remission at the end of } \\
\text { study (on average } 7 \text { th week) }\end{array}$ \\
\hline Schutter (2010) & 9 & $9(3.57)$ & $50.0(6.3)$ & $\begin{array}{l}d=0.63 \text { for EOT mean change in severity with no difference between } \\
\text { left and right frontal sites; trials using LF rTMS }\end{array}$ \\
\hline Slotema et al (2010) & 40 & $10.45(3.33)$ & Not presented & $\begin{array}{l}d=0.55 \text { based on } 34 \text { rTMS } v \text {. sham RCTs; } d=0.53 \text { for left, } d=0.84 \text { for } \\
\text { right and } d=0.47 \text { for bilateral frontal sites }\end{array}$ \\
\hline
\end{tabular}

d, Cohen's $d$ effect size; EOT, end of treatment session (actual time dependent on number of sessions administered, usually over 2-4 weeks); HF, high frequency; LF, low frequency; NNT, number needed to treat: RCT, randomised controlled trial; remission, post-treatment 17-item or 21-item Hamilton Rating Scale for Depression scores not above 7 or 8, respectively, or Montgomery-Åsberg Depression Rating Scale scores not above 6; response, at least $50 \%$ reduction in post-treatment scores on accepted depression rating scales; rTMS, repetitive transcranial magnetic stimulation; TRD, treatment-resistant depression; WMD, weighted mean difference.

a. Note of caution: all of these studies were of short duration; the durations of individual studies were the same as the length of rTMS treatment in most cases.

b. s.d. not provided.

c. Mean and s.d. not provided.

Source: adapted from Sabesan et al (2015). 
have shown it to be effective as an augmentation therapy or as a monotherapy with a benign tolerability profile (Berlim 2013a,b,c; Gross 2007; Schutter 2009). The initial device approval by the FDA was based on a post hoc analysis that focused on patients with one antidepressant trial failure (that is, with a low level of treatment resistance as defined by Thase $\&$ Rush, 1997). However, meta-analysis of RCTs that specifically focused on patients with more than two antidepressant trial failures (range 3.2-6.5, where data were available) suggests that rTMS produces three times higher short-term response rates when compared with sham treatment and five times higher short-term remission rates (Gaynes 2014). At present, it is uncertain how long these benefits last.

Durability studies have reported a 13\% relapse rate when participants are followed up for 6 months. In one particular study (Janicak 2010), 301 patients with treatment-resistant depression were randomised to active or sham TMS for 6 weeks, followed by a 6 -week open-label trial (in which both the researchers and participants knew which treatment was being administered) involving participants who did not respond in the initial study. Participants were followed up while being maintained on protocol-based antidepressant monotherapy, with 'rescue TMS' if required. Thirty-eight per cent of participants required rescue TMS for symptom worsening; of these, $84 \%$ recovered with the rescue administrations. Overall, 10\% relapsed despite this combined TMS and pharmacotherapy approach.

Longitudinal studies with 1-year follow-up data showed a $37 \%$ relapse rate among those who initially responded or remitted with acute rTMS treatment (Dunner 2014). Sixty-two per cent of the 49 patients who received maintenance rTMS for 6 months after their initial response to acute rTMS treatment continued to maintain their responder status (Connolly 2012). However, owing to the paucity of evidence supporting the longterm efficacy of rTMS, we consider the current evidence to be limited to short-term efficacy and not applicable to relapse prevention (Fig. 1). Approaches such as episodic 'rescue TMS' appear promising, but they do not have experimental support at this stage.

\section{Safety}

The international scientific community has evaluated the available evidence on the administration of TMS in both clinical and research settings and has developed a safety protocol (Rossi 2009). The potential side-effects of TMS include seizure induction, transient acute hypomania,

\begin{tabular}{|c|c|}
\hline $\begin{array}{ll}\text { TABLE } 2 & \text { Report } \\
\text { transcr }\end{array}$ & $\begin{array}{l}\text { Iverse effects of repetitive } \\
\text { I magnetic stimulation (rTMS) }\end{array}$ \\
\hline Adverse effect & $\begin{array}{c}\text { Frequency with most commonly } \\
\text { used rTMS protocols }\end{array}$ \\
\hline Headache & $9.7 \%$ \\
\hline Scalp discomfort & $9.3 \%$ \\
\hline Facial twitches & $1.9 \%$ \\
\hline Lacrimation & $1.5 \%$ \\
\hline Local redness & $1.3 \%$ \\
\hline Drowsiness & $2.5 \%$ \\
\hline
\end{tabular}

Source: adapted from Slotema et al (2010).

syncope, transient headache, neck pain and local discomfort, transient hearing changes and transient cognitive changes. A review of the most recent evidence on rTMS for sensory phenomena concluded that TMS is generally well tolerated by patients (Muller 2012). A summary of key adverse effects from meta-analysis of rTMS studies is reported by Slotema et al (2010) (Table 2).

\section{Patients' experience of rTMS}

Little has been published about patients' actual experience of rTMS. Rosedale et al (2009) conducted a phenomenological study and found that a certain mindfulness emerged during the treatment process. Participants reported being conscious of what they were thinking, and they began to imagine themselves in different situations with new possibilities being realised. The participants in this study 'consistently reinforced that connection with the clinician influenced treatment experience, completion, and depression outcomes'. They reported that their relationship with the clinician was strengthened when time was allotted to discuss the mechanism of TMS, to explain stimulus placement, to show concern about comfort and clinical response, and to demonstrate interest in the participant's ongoing life; and also when the clinician was available for emergencies between sessions.

\section{Availability of TMS in the NHS}

At present, TMS units are being set up in many National Health Service (NHS) trusts. TMS clinics are already in operation in Nottingham (supported by Nottinghamshire Healthcare NHS Foundation Trust), Northampton (supported by Northamptonshire Healthcare NHS Foundation Trust) and Grimsby (supported by NAVIGO, the local mental healthcare provider). TMS treatment for depression can also be obtained from several private providers, especially in London. Figure 1 illustrates the place for TMS 


\section{MCQ answers}

$1 d \quad 2 b \quad 3 c \quad 4$ e $\quad 5 b$ in the current depression treatment pathway. This pathway contrasts with most of the rTMS studies, which have typically been carried out in people who have failed to respond to only one antidepressant treatment trial.

\section{Conclusions}

Neuromodulation is an emerging new therapeutic field for the treatment of neuropsychiatric conditions. During the past decade, TMS has been used widely for the treatment of depression and is now an established, safe and effective option for treatment-resistant depression. There is a need for large, well-conducted, longer-term RCTs of TMS in populations of patients who have failed to respond to three or four antidepressants and psychological therapies, in addition to pragmatic evaluation of its utility in the less treatment-resistant populations in which most RCTs have been conducted to date.

\section{References}

Allan C, Herrmann L, Ebmeier K (2011) Transcranial magnetic stimulation in the management of mood disorders. Neuropsychobiology, 64: 163-9.

American Psychiatric Association (2013) The Diagnostic and Statistical Manual of Mental Disorders (5th edn) (DSM-5). APA.

Barker A, Jalinous R, Freeson I (1985) Non-invasive magnetic stimulation of the human motor cortex. Lancet, 1: 1106-7.

Bauer M, Whybrow PC, Angst J, et al (2002) World Federation of Societies of Biological Psychiatry (WFSBP) Guidelines for Biological Treatment of Unipolar Depressive Disorders, Part 1: Acute and continuation treatment of major depressive disorder. World Journal of Biological Psychiatry, 3: 5-43.

Berlim M, Van den Eynde F, Daskalakis Z (2013a) A systematic review and meta-analysis on the efficacy and acceptability of bilateral repetitive transcranial magnetic stimulation (rTMS) for treating major depression. Psychological Medicine, 43: 2245-54

Berlim M, Van den Eynde F, Daskalakis Z (2013b) Efficacy and acceptability of high frequency repetitive transcranial magnetic stimulation (rTMS) versus electroconvulsive therapy (ECT) for major depression: a systematic review and meta-analysis of randomized trials. Depression and Anxiety, 30: 614-23.

Berlim M, Van den Eynde F, Daskalakis ZJ (2013c) Clinically meaningful efficacy and acceptability of low-frequency repetitive transcranial magnetic stimulation (rTMS) for treating primary major depression: a meta-analysis of randomized, double-blind and sham-controlled trials. Neuropsychopharmacology, 38: 543-51.

Berlim MT, Van den Eynde F, Daskalakis ZJ (2013d) High-frequency repetitive transcranial magnetic stimulation accelerates and enhances the clinical response to antidepressants in major depression: a metaanalysis of randomized, double-blind, and sham-controlled trials. Journal of Clinical Psychiatry, 74: 122-9.

Connolly K, Helmer A, Cristancho M, et al (2012) Effectiveness of transcranial magnetic stimulation in clinical practice post-FDA approval in the United States: results observed with the first 100 consecutive cases of depression at an academic medical center. Journal of Clinical Psychiatry, 73: 567-73.

Couturier J (2005) Efficacy of rapid-rate repetitive transcrania magnetic stimulation in the treatment of depression: a systematic review and meta-analysis. Journal of Psychiatry \& Neuroscience, 30 : 83-90.

Dunner D, Aaronson S, Sackeim H, et al (2014) A multisite, naturalistic, observational study of transcranial magnetic stimulation for patients with pharmacoresistant major depressive disorder: durability of benefit over a 1-year follow-up period. Journal of Clinical Psychiatry, 75: 1394-401.

Fitzgerald P, Fountain S, Daskalakis Z (2006) A comprehensive review of the effects of rTMS on motor cortical excitability and inhibition. Clinical Neurophysiology, 117: 2584-96.

Fitzgerald P, Daskalakis Z (2013) Repetitive Transcranial Magnetic Stimulation Treatment for Depressive Disorders: A Practical Guide. Springer-Verlag.

Furukawa T (2014) How can we make the results of trials and their meta-analyses using continuous outcomes clinically interpretable? Acta Psychiatrica Scandinavica, 130: 321-3.

Gaynes BN, Lloyd SW, Lux L, et al (2014) Repetitive transcranial magnetic stimulation for treatment-resistant depression: a systematic review and meta-analysis. Journal of Clinical Psychiatry, 75: 477-89.

Groppa S, Oliviero A, Eisen A, et al (2012) A practical guide to diagnostic transcranial magnetic stimulation: report of an IFCN committee. Clinical Neurophysiology, 123: 858-82.

Gross M, Nakamura L, Pascual-Leone A, et al (2007) Has repetitive transcranial magnetic stimulation (rTMS) treatment for depression improved? A systematic review and meta-analysis comparing the recent vs. the earlier rTMS studies. Acta Psychiatrica Scandinavica, 116: $165-73$

Herrmann L, Ebmeier K (2006) Factors modifying the efficacy of transcranial magnetic stimulation in the treatment of depression: a review. Journal of Clinical Psychiatry, 67: 1870-6.

Hoogendam J, Ramakers GM, Di Lazzaro V (2010) Physiology of repetitive transcranial magnetic stimulation of the human brain. Brain Stimulation, 3: 95-118.

Horvath J, Mathews J, Demitrack M, et al (2010) The NeuroStar TMS device: conducting the FDA approved protocol for treatment of depression. Journal of Visualized Experiments, 45: e2345.

Janicak P, Nahas Z, Lisanby S, et al (2010) Durability of clinical benefit with transcranial magnetic stimulation (TMS) in the treatment of pharmacoresistant major depression: assessment of relapse during a 6-month, multisite, open-label study. Brain Stimulation, 3: 187-99.

Kedzior K, Azorina V, Reitz S (2014) More female patients and fewer stimuli per session are associated with the short-term antidepressant properties of repetitive transcranial magnetic stimulation (rTMS): a meta-analysis of 54 sham-controlled studies published between 1997-2013. Neuropsychiatric Disease and Treatment, 10: 727-56.

Kito S, Fujita K, Koga Y (2008) Regional cerebral blood flow changes after low-frequency transcranial magnetic stimulation of the right dorsolateral prefrontal cortex in treatment-resistant depression. Neuropsychobiology, 58: 29-36.

Krstic J, Buzadžic I, Milanovic S, et al (2014) Low-frequency repetitive transcranial magnetic stimulation in the right prefrontal cortex combined with partial sleep deprivation in treatment-resistant depression: a randomized sham-controlled trial. Journal of ECT, 30 : $325-31$

Lam R, Chan P, Wilkins-Ho M, et al (2008) Repetitive transcranial magnetic stimulation for treatment-resistant depression: a systematic review and meta-analysis. Canadian Journal of Psychiatry, 53: 621-31.

Lepping P, Schönfeldt-Lecuona C, Sambhi R, et al (2014) A systematic review of the clinical relevance of repetitive transcranial magnetic stimulation. Acta Psychiatrica Scandinavica, 130: 326-41.

Luscher C, Malenka R (2012) NMDA receptor-dependent long-term potentiation and long-term depression (LTP/LTD). Cold Spring Harbor Perspectives in Biology, 4: a005710.

Martin JL, Barbanoj MJ, Schlaepfer TE, et al (2003) Repetitive transcranial magnetic stimulation for the treatment of depression. Systematic review and meta-analysis. British Journal of Psychiatry, 182: 480-91.

Mitchell P, Loo C (2006) Transcranial magnetic stimulation for depression. Australian and New Zealand Journal of Psychiatry, 40: 406-13. 
Muller P, Pascual-Leone A, Rotenberga A (2012) Safety and tolerability of repetitive transcranial magnetic stimulation in patients with pathologic positive sensory phenomena: a review of literature. Brain Stimulation, 5: 320-9

NHS Evidence (2012) Depression: Evidence Update April 2012 (Evidence Update 13). National Institute for Health and Care Excellence.

National Institute for Health and Care Excellence (2007) Transcranial Magnetic Stimulation for Severe Depression (IPG242). NICE.

National Institute for Health and Care Excellence (2015a) P346/2 Repetitive transcranial magnetic stimulation for depression Consultation Comments Table. NICE (https://www.nice.org.uk/guidance/IPG542/ documents/consultation-comments-and-responses). Accessed 23 September 2016

National Institute for Health and Care Excellence (2015b) Repetitive Transcranial Magnetic Stimulation for Depression (IPG542). NICE.

Ren J, Li H, Palaniyappan L, et al (2014) Repetitive transcranial magnetic stimulation versus electroconvulsive therapy for major depression: a systematic review and meta-analysis. Progress in NeuroPsychopharmacology and Biological Psychiatry, 51: 181-9.

Rosedale M, Lisanby S, Malaspina D (2009) The structure of the lived experience for persons having undergone rTMS for depression treatment. Journal of the American Psychiatric Nurses Association, 15: 333-7.

Rossi S, Hallett M, Rossini P, et al (2009) Safety, ethical considerations, and application guidelines for the use of transcranial magnetic stimulation in clinical practice and research. Clinical Neurophysiology, 120: $2008-39$
Sabesan P, Lankappa S, Khalifa N, et al (2015) Transcranial magnetic stimulation for geriatric depression: promises and pitfalls. World Journal of Psychiatry, 5: 170-81.

Salomons T, Dunlop K, Kennedy S, et al (2014) Resting-state corticothalamic-striatal connectivity predicts response to dorsomedial prefrontal rTMS in major depressive disorder. Neuropsychopharmacology, 39: 488-98.

Schutter D (2009) Antidepressant efficacy of high-frequency transcranial magnetic stimulation over the left dorsolateral prefrontal cortex in double-blind sham-controlled designs: a meta-analysis. Psychological Medicine, 39: 65-75.

Schutter D (2010) Quantitative review of the efficacy of slow-frequency magnetic brain stimulation in major depressive disorder. Psychological Medicine, 40: 1789-95

Slotema CW, Blom JD, Hoek HW, et al (2010) Should we expand the toolbox of psychiatric treatment methods to include repetitive transcranial magnetic stimulation (rTMS)? A meta-analysis of the efficacy of rTMS in psychiatric disorders. Journal of Clinical Psychiatry, 71: 873-84.

Tang 0, Li G, Wang A, et al (2015) A systematic review for the antidepressant effects of sleep deprivation with repetitive transcranial magnetic stimulation. BMC Psychiatry, 15: 1-9.

Thase M, Rush A (1997) When at first you don't succeed: sequential strategies for antidepressant nonresponders. Journal of Clinical Psychiatry, 58 (suppl 13): 23-9.

\section{MCOs}

Select the single best option for each question stem

\section{According to the most recent NICE} guidance for rTMS in depression:

a rTMS can be used for any type of depression

b rTMS should not be combined with pharmacotherapy

c rTMS is best used as an adjunct to ECT

d rTMS has adequate evidence regarding safety and short-term efficacy

e rTMS should only be used in research settings.

2 The most likely mechanism by which rTMS works in the treatment of depression is:

a providing regular contact with a healthcare professional

b directly stimulating areas of the brain relevant to depression

$c$ inducing involuntary muscle movements

d improving sleep

e stimulating neurons to become stronger.

\section{A contraindication for rTMS as a treatment} for depression is:

a pregnancy

b presence of metal fillings

c presence of a cochlear implant

d asthma

e diabetes.

4 The administration of TMS requires expertise in:

a neuroanatomy

$\mathrm{b}$ anaesthetic administration

c interpretation of EEG

$d$ interpretation of ECG

e estimating motor threshold.

\author{
5 A common side-effect of rTMS is: \\ a joint pain \\ b neck pain \\ c blurred vision \\ d unusual behaviour \\ e tiredness.
}

